\title{
Relationship between vitamin D levels and platelet count: A retrospective study
}

\author{
(1) Recep Alanlı, (1) Murat Bülent Küçükay, (1) Kadir Serkan Yalçın \\ Lokman Hekim University Faculty of Medicine, Ankara Hospital, Clinic of Internal Medicine, Ankara, Turkey
}

\section{Date submitted:}

08.11.2019

Date accepted:

27.04.2020

Online publication date:

15.09.2020

\section{Corresponding Author:}

Recep Alanlı, MD, Lokman Hekim University Faculty of Medicine, Ankara Hospital, Clinic of Internal Medicine, Ankara, Turkey

recepalanli@gmail.com

ORCID:

orcid.org/0000-0003-4663-1898

Keywords: Platelet count, mean platelet volume, vitamin $\mathrm{D}$

\begin{abstract}
Aims: Vitamin D deficiency, increase in mean platelet volume (MPV) and high platelet counts are related to several diseases. The aim of this study is to analyze the relationship between vitamin D status and platelet count, MPV, age, gender, alkaline phosphatase (ALP), and uric acid on patients.

Methods: This study included 899 patients, who did not have any chronic diseases and conditions that affected platelet counts. Calcium, magnesium, ALP, uric acid, 25-hydroxyvitamin D3 levels and 22 parameter hemogram; including MPV and platelet counts were analyzed. Participants were separated into three groups according to their 25-hydroxyvitamin D3 levels: serious deficiency (24.96 nmol/L), deficiency (24.96-49.92 nmol/L), and insufficiency (49.92-74.88 $\mathrm{nmol} / \mathrm{L})$. The difference among the groups were analyzed by using the one-way ANOVA test. The Pearson's correlation analysis was used to evaluate associations between 25-hydroxyvitamin D3 levels and other variables.
\end{abstract}

Results: There was a significant negative correlation between 25-hydroxyvitamin D3 levels and platelet counts in groups $(p=0.001, r=-0.108)$. The mean vitamin D level was $38.19 \pm 17.47 \mathrm{nmol} / \mathrm{L}$ in female participants and $42.68 \pm 12.48 \mathrm{nmol} / \mathrm{L}$ in males $(\mathrm{p}<0.001)$. A significant difference between platelet counts in serious vitamin D deficiency $(286 \pm 68 \times 10 \% / L)$ and insufficiency groups $(268 \pm 64 \times 109 / L)$ was also noted $(p=0.008)$.

Conclusions: There was a negative correlation between 25-hydroxyvitamin D3 levels and platelet counts in all groups. Low levels of 25-hydroxyvitamin D3 were associated with an increased platelet count in participants.

\section{Introduction}

Platelets are basic components in primary hemostasis, immune system, and wound healing (1). Thrombopoiesis and platelet apoptosis determine actual platelet count. When there are no affecting conditions, platelet counts are usually stable at bloodstream. Platelet count may decrease in older ages. Gender, ethnicity, and environmental factors may affect platelet counts (2).

Mean platelet volume (MPV) reflects platelet size and is a marker of platelet function (3). Higher MPV levels may reflect the existence of large platelets which have higher aggregation tendency. Large platelets are found to be more active and thrombogenic compared to small platelets (4). MPV levels are increased in obesity, acute myocardial infarction, hyperlipidemia, hypertension, stroke, preeclampsia and renal artery stenosis (5). Platelet hyperactivity is a risk factor for coronary artery disease
(CAD) and increased MPV was found to be associated with platelet hyperactivity (6). High MPV levels were associated with increased mortality in CAD (7). Also, MPV has been reported to be a risk factor for CAD and acute myocardial infarction in hemodialysis patients (8).

Vitamin D is synthesized from cholesterol and has hormonal activity. Vitamin D has some important metabolic effects such as regulation of calcium and phosphorus homeostasis, bone mineralization, enhancing immune system, regulating cell division and differentiation, regulating coagulation and decreasing inflammation. Vitamin $D$ enhances calcium absorption in the duodenum and reduces calcium excretion by the kidney (9). Low vitamin $D$ levels were associated with coronary heart disease, metabolic syndrome, insulin resistance, susceptibility to infections, allergic diseases, malignancies and autoimmune diseases (10). 
The active form of vitamin $D$ is 1,25 dihydroxyvitamin $D$. 25-hydroxyvitamin $D$ is the major circulating form of vitamin $\mathrm{D}$ and is converted to 1.25 dihydroxyvitamin D mainly at the kidneys. 25-hydroxyvitamin $\mathrm{D}$ level in body is almost thousand fold compared to 1.25 dihydroxyvitamin $\mathrm{D}$ level. While 25-hydroxyvitamin $D$ has a half-life of two to three weeks, 1.25 dihydroxyvitamin $D$ has only four to six hours half-life. The best indicator of vitamin $D$ status is the serum 25-hydroxyvitamin $D$ concentration because it reflects both dietary intake from vitamin $D$ and cutaneous synthesis of vitamin D (11).

Magnesium deficiency causes decreased production of vitamin D (12). Decreased calcium intake with diet may cause lower vitamin $\mathrm{D}$ levels and hypocalcemia decreases half-life of vitamin D (13).

Previous studies about the relationship between vitamin $D$ and age, gender, platelet count, uric acid and alkaline phosphatase (ALP) demonstrated controversial results. The effect of calcium and magnesium serum levels on vitamin $D$ metabolism was not considered. Taking this into account, this study was planned to inspect people who had normal serum calcium and magnesium levels.

The purpose of this study is to analyze the correlation between vitamin D deficiency and platelet count, MPV, age, gender, ALP, and uric acid on participants with normal calcium and magnesium levels.

\section{Methods}

This is a single-center, retrospective study conducted in an affiliated foundation hospital in Ankara. This study was approved by Lokman Hekim University Ethical Committee (2019/51). This study was carried out in accordance with The Code of Ethics of the World Medical Association (Declaration of Helsinki) for experiments involving humans (link: https://www.wma.net/ policies-post/wma-international-code-of-medical-ethics/), and Uniform Requirements for manuscripts submitted to Biomedical journals (link: http://www.icmje.org/).

From the people admitted to internal medicine department between January 1, 2018 and December 31, 2018, a total of 899 were enrolled. Participants who had vitamin D level less than $74.88 \mathrm{nmol} / \mathrm{L}$, normal levels of calcium and magnesium were included in the study. In order to prevent other factors affecting platelet count, some diseases and conditions were accepted as exclusion criteria. People under 15 years of age (since they were admitted to pediatrics clinics), people who had previous history of CAD, chronic obstructive pulmonary disease, stroke, chronic liver disease, chronic kidney disease (estimated GFR<60 mL/min), malignancy, calcium metabolism disorders and acute infectious conditions were not included in the study. People using drugs affecting platelet counts, such as angiotensin-converting enzyme inhibitors, anticoagulants, acetylsalicylic acid, non-steroidal anti-inflammatory drugs were not included in the study.

Blood samples were obtained after 12 hours of fasting at admission and were analyzed in the laboratory without delay. Magnesium, calcium, ALP, and uric acid tests were analyzed by Roche Hitachi Cobas 501 (Switzerland). Platelet count and MPV were analyzed using XN-1000 analyzer (USA) with 22 parameters. 25-hydroxyvitamin $D$ was measured by highpressure liquid chromatography on the Roche Hitachi Cobas 601 (Switzerland) analyzer. According to Turkey Society of Endocrinology and Metabolism "Osteoporosis and Metabolic Bone Diseases Diagnosis Treatment Guidelines" patients were divided into three groups based on their vitamin $D$ levels: serious deficiency $24.96 \mathrm{nmol} / \mathrm{L})$, deficiency (24.96-49.92 nmol/L), and insufficiency $(49.92-74.88 \mathrm{nmol} / \mathrm{L})(14)$.

\section{Statistical Analysis}

All data were evaluated using the statistical software SPSS for Windows (version 25, SPSS, Chiago, IL, USA). All variables were checked with the Kolmogorov-Smirnov normality test. Variables in all groups showed a normal distribution. Using the one-way ANOVA test, these groups were analyzed. The Pearson's correlation analysis was performed to evaluate the associations between vitamin $D$ and other variables. $P$ value smaller than 0.05 was considered to be statistically significant.

\section{Results}

There was a significant negative correlation between 25-hydroxyvitamin D3 levels and platelet counts in groups $(p=0.001, r=-0.108)$. Increased platelet counts were found in people with low vitamin D levels. However, there was no significant correlation between vitamin $\mathrm{D}$ and age, gender, uric acid, MPV, and ALP in vitamin D groups. Significant difference between platelet counts in serious vitamin $D$ deficiency $(286 \pm 68 \times 109 / L)$ and insufficiency groups $(268 \pm 64 \times 109 / L)$ was also noted $(p=0.008)$. In the serious vitamin $D$ deficient group, platelet counts were significantly higher than in other groups. Platelet counts according to vitamin D groups are shown in Figure 1.

The mean vitamin $D$ level was $38.19 \pm 17.47 \mathrm{nmol} / \mathrm{L}$ in female participants and $42.68 \pm 12.48 \mathrm{nmol} / \mathrm{L}$ in males $(p<0.001)$. Compared to male participants, vitamin $D$ levels were lower in female participants in all groups.

Participants were between 15 and 70 years old, 585 (65.1\%) were female and 314 (34.9\%) were male. The mean age of participants was $40.3 \pm 14.3$ years. Male participants' mean age was $41.4 \pm 14$ years. Female participants' mean age was $40.3 \pm 14$ years.

Characteristics of subjects and laboratory parameters according to vitamin $\mathrm{D}$ groups are shown in Table 1. 


\section{Discussion}

Vitamin $\mathrm{D}$ deficiency is a problem all over the world and one billion people suffer from vitamin D deficiency (11). Vitamin D deficiency is also very common in Turkey. In Turkish Diabetes, Hypertension, Obesity and Endocrinological Diseases Prevalence Study-II, 9560 adults were examined and it was determined that $93 \%$ of participants' vitamin D levels were lower than $49.92 \mathrm{nmol} / \mathrm{L}$ (15). In another study with 35.667 participants, the mean serum vitamin D level was $36.19 \mathrm{nmol} / \mathrm{L}$ among females and $45.18 \mathrm{nmol} / \mathrm{L}$ among males. Also $94.4 \%$ of the participants' serum vitamin D levels were less than 74.88 $\mathrm{nmol} / \mathrm{L}(16)$. In this reported study, the mean vitamin $D$ level was $38.19 \pm 17.47 \mathrm{nmol} / \mathrm{L}$ in females and $42.68 \pm 12.48 \mathrm{nmol} / \mathrm{L}$ in males. All of our participants' vitamin $D$ levels were less than $74.88 \mathrm{nmol} / \mathrm{L}$.

Vitamin D levels of people may vary according to seasons because of the angle of the sunlight reaching the Earth changes. Santos et al. (17) reported that vitamin D levels were highest in August and lowest in March. In order to minimize seasonal changes that could affect the results, participants admitted in the whole year from beginning of January to the end of December were enrolled in this study.

In one study by Hovsepian et al. (18), it was demonstrated that vitamin $D$ levels in female participants were significantly

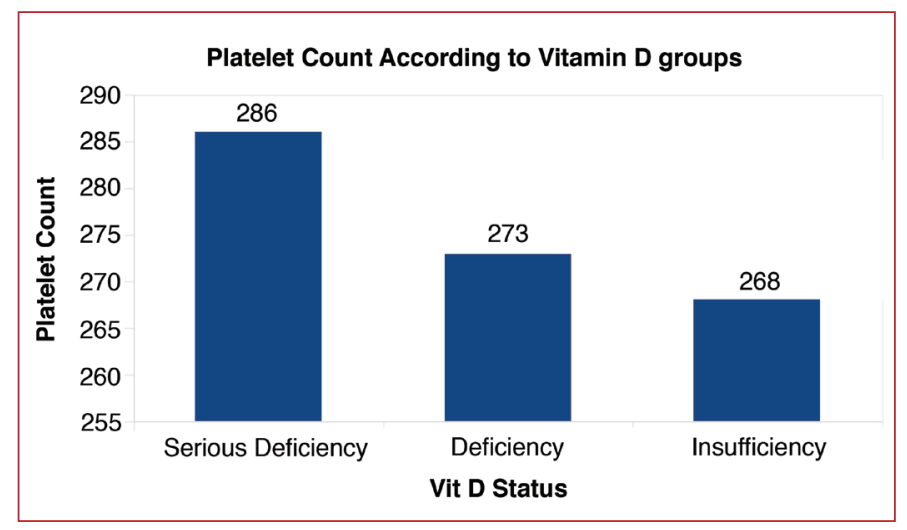

Figure 1. Platelet counts $(x 109 / \mathrm{L})$ according to vitamin $\mathrm{D}$ groups lower. In another study with 5531 participants who did not have any chronic disease, female participants had lower vitamin D levels than males (19). Compared to male population, female population have more sedentary lifestyle, spend more time indoors and use sunscreen creams more often; therefore, they do not get efficient benefit of sunlight. Consistent with other studies in the literature, the mean vitamin D level was lower in females in this reported study.

In one study with 78 participants, there was a significant negative relationship between vitamin $D$ level and MPV in CAD patients (20). In another study, there was a negative relationship between vitamin D levels and MPV levels in gestational diabetes mellitus (21). Cumhur et al. (22) reported that there was no significant relationship between vitamin $D$ deficiency and MPV levels on 434 healthy participants. These controversial results have been reported to be caused by the differences in measurement techniques, waiting time after obtaining blood samples, anticoagulants used in tubes that blood samples were stored. Also, there was no standardization in analysis method. All these factors may change MPV levels (23). In this reported study, there was no significant correlation between vitamin $D$ levels and MPV levels.

There was a significant negative correlation between vitamin $D$ levels and platelet counts in a study reported from Korea. This was explained by anti-thrombogenic, antiinflammatory, antioxidant and anticoagulant activity of vitamin D (9). Aihara et al. (24) reported a study with vitamin D receptor (VDR) knock-out mice. In order to incite thrombosis, lipopolysaccharide injections were performed. Although calcium levels were divergent, thrombosis developed in many different tissues in these mice. They concluded that VDR system had an important role in anti-thrombogenicity in vivo (2). VDRs were found in mitochondria of thrombocytes and by these receptors vitamin $D$ regulates protein synthesis and thrombocyte functions (25). Megakaryocytes are precursors for thrombocytes and they also have VDRs. The stimulation of these receptors regulates cell maturation and megakaryocyte

\begin{tabular}{|c|c|c|c|c|}
\hline & Serious deficiency & Deficiency & Insufficiency & $p$ value \\
\hline Age (y) & $39.5 \pm 15.5$ & $39.8 \pm 13.5$ & $41.7 \pm 13.8$ & 0.19 \\
\hline Gender (female/male) (\%) & $86.2 / 13.8$ & $61.2 / 38.8$ & $68.8 / 31.2$ & $<0.001$ \\
\hline ALP $(\mu k a t / L)$ & $1.27 \pm 0.61$ & $1.24 \pm 0.54$ & $1.25 \pm 0.62$ & 0.9 \\
\hline MPV (fL) & $10 \pm 0.7$ & $10 \pm 0.9$ & $9.9 \pm 0.8$ & 0.38 \\
\hline Platelet count $(x 109 / \mathrm{L})$ & $286.1 \pm 68$ & $273.2 \pm 77.8$ & $268.1 \pm 64.5$ & 0.04 \\
\hline Uric acid $(\mu \mathrm{mol} / \mathrm{L})$ & $279.58 \pm 83.28$ & $291.48 \pm 89.23$ & $297.43 \pm 77.33$ & 0.36 \\
\hline Calcium (mmol/L) & $2.35 \pm 0.07$ & $2.35 \pm 0.10$ & $2.38 \pm 0.05$ & 0.06 \\
\hline Magnesium (mmol/L) & $1.0 \pm 0.05$ & $1.0 \pm 0.05$ & $1.0 \pm 0.05$ & 0.44 \\
\hline $25(\mathrm{OH})$ vitamin D3 $(\mathrm{nmol} / \mathrm{L})$ & $17.22 \pm 4.74$ & $37.69 \pm 6.99$ & $60.65 \pm 7.24$ & $<0.001$ \\
\hline
\end{tabular}


proliferation. Besides, 1.25 dihydroxyvitamin $\mathrm{D}$ is reported to downregulate VDR (26). Vitamin D deficiency promotes megakaryocyte maturation and increases thrombocyte counts (25).

It was documented that vitamin $\mathrm{D}$, itself, has antioxidant activity (27). A study reported an association between oxidative stress and platelet count (28). In another study, antioxidant consumption resulted in lower platelet counts (29). Oxidative stress is related with higher platelet counts (30). Interleukin-6 (IL-6) is an inflammatory cytokine and elevated IL-6 levels results in increased oxidative stress and promotes megakaryocyte production and maturation (31). There was an inverse correlation between vitamin D and IL-6 levels in a study on 1381 healthy participants (32). After all these findings, lower vitamin $D$ levels were thought to be related with inflammation and elevated cytokine levels that would result in increased thrombocyte count.

Vitamin D suppresses vascular cell adhesion molecule-1 and membrane type 1 matrix-metalloproteinase expression and platelet activation, fibrinolysis and thrombosis will be decreased and endothelium will be preserved accordingly (33). Vitamin D insufficiency will cause lower nitric oxide levels (25). As a result, endothelial dysfunction develops in vitamin D insufficiency and this causes increased platelet activation and thrombosis (33). It has been thought that all these mechanisms caused increased thrombocyte production. In this reported study, there was a significant negative correlation between vitamin $D$ levels and platelet counts.

Limitations of this study should be reported include that this was a single-center and retrospective study. The data were limited to the recorded information. Participants included in this study were people who were admitted to hospital and data from healthy population were not obtained, thus results of this reported study may not be generalized to the entire population.

\section{Conclusion}

In conclusion, there was no previous study which reported a relationship between platelet counts and vitamin $D$ levels in patients whose serum calcium and magnesium levels were normal. According to the results of this study, lower vitamin D levels will cause higher platelet counts in people with normal calcium and magnesium levels. In literature, there is only one study that reported a significant correlation between vitamin $\mathrm{D}$ levels and platelet counts previously and this reported study will be the second. Elevated thrombocyte counts in vitamin $\mathrm{D}$ deficiency was thought to be caused by increased inflammatory cytokines and endothelial dysfunction. In patients who have thrombotic event risks, vitamin D supplementation may decrease thrombocytosis and development of thrombotic event. Hemogram is a basic test that can be analyzed everywhere. Increase in platelet count may reflect vitamin D deficiency in suspected people. This may be helpful in the diagnosis of vitamin $D$ deficiency, where vitamin $D$ levels cannot be studied.

\section{Acknowledgement}

Authors of this study thank to Mr. Fatih Acikgoz for statistical analysis and advices and Dr. Gohar Seyedi for editing support.

\section{Ethics}

Ethics Committee Approval: This study was approved by Lokman Hekim University Ethical Committee (2019/51).

Informed Consent: Retrospective study.

Peer-review: Externally and internally peer-reviewed.

\section{Authorship Contributions}

Concept: R.A., Design: R.A., M.B.K., K.S.Y., Data Collection or Processing: R.A., M.B.K., K.S.Y., Analysis or Interpretation: R.A., M.B.K., K.S.Y., Literature Search: R.A., M.B.K., Writing: R.A., M.B.K.

Conflict of Interest: No conflict of interest was declared by the authors.

Financial Disclosure: The authors declared that this study received no financial support.

\section{References}

1. Patzelt J, Langer HF. Platelets in angiogenesis. Curr Vasc Pharmacol. 2012;10:570-577.

2. Ittermann T, Feig MA, Petersmann A, et al. Mean platelet volume is more important than age for defining reference intervals of platelet counts. Plos One. 2019;14:e0213658.

3. Davi G, Patrono C. Platelet activation and atherothrombosis. N Engl J Med. 2007;357:2482-2494.

4. Rafieian-Kopaie M, Nasri H. Platelet counts and mean platelet volume in association with serum magnesium in maintenance hemodialysis patients. J Renal Inj Prev. 2012;1:17-21.

5. Yazici M, Kaya A, Kaya Y, Albayrak S, Cinemre H, Ozhan H. Lifestyle modification decreases the mean platelet volume in prehypertensive patients. Platelets. 2009;20:58-63.

6. Ozdemir O, Soylu M, Alyan O, et al. Association between mean platelet volume and autonomic nervous system functions: Increased mean platelet volume reflects sympathetic over activity. Exp Clin Cardiol. 2004;9:243247.

7. Sansanaydhu N, Numthavaj P, Muntham D, et al. Prognostic effect of mean platelet volume in patients with coronary artery disease. A systematic review and metaanalysis. Thromb Haemost. 2015;114:1299-1309.

8. Henning BF, Zidek W, Linder B, Tepel M. Mean platelet volume and coronary heart disease in hemodialysis patients. Kidney Blood Press Res. 2002;25:103-108. 
9. Park YC, Kim J, Seo MS, Hong SW, Cho ES, Kim JK. Inverse relationship between vitamin D levels and platelet indices in Korean adults. Hametology. 2017;22:623-629.

10. Akbas EM, Gungor A, Ozcicek A, Akbas N, Askin S, Polat $M$. Vitamin $D$ and inflammation: evaluation with neutrophil to-lymphocyte ratio and platelet-to-lymphocyte ratio. Arch Med Sci. 2016;12:721-727.

11. Holick MF. Vitamin D Deficiency. N Engl J Med. 2007;357:266-281.

12. Risco F, Traba ML. Influence of magnesium on the in vitro synthesis of 24,25-dihydroxyvitamin D3 and 1 alpha, 25-dihydroxyvitamin D3. Magnes Res. 1992;5:5-14.

13. Lips P. Interaction Between Vitamin D and Calcium. Scan J Clin Lab Invest Suppl. 2012;243:60-64.

14. Turkish Society of Endocrinology and Metabolism. Guide to Metabolic Bone Diseases. Accessed on date: April 9, 2019. Available from: http://temd.org.tr/admin/uploads/ tbl_kilavuz/METABOLIK_KH_BOOK_web_.pdf

15. Satman I, Turkey Society of Endocrinology and Metabolism Results of TURDEP-II study. Accessed on date: April 17, 2019. Available from: http://www.turkendokrin.org/files/file/ TURDEP_II_2011.pdf

16. Solak I, Cihan FG, Mercan S, KethudaT, Eryilmaz MA. Evaluation of 25-Hydroxyvitamin D Levels in Central Anatolia, Turkey. Biomed Res Int. 2018;2018:4076548.

17. Santos MJ, Fernandes V, Garcia FM. Vitamin D Insufficiency in a Hospital Population: A Photograph from the Laboratory Perspective. Acta Med Port. 2015;28:726-734.

18. Hovsepian S, Amini M, Aminorroaya A, Amini P, Iraj P. Prevalence of vitamin $D$ deficiency among adult population of Isfahan City, Iran. J Health Popul Nutr. 2011;29:149-155.

19. Ning Z, Song S, Miao L, et al. High prevalence of vitamin $D$ deficiency in urban health checkup population. Clin Nutr. 2016;35:859-863.

20. Szlacheta IK, Hudzik B, Nowak J, et al. Mean platelet volume is associated with serum 25-hydroxyvitamin D concentrations in patients with stable coronary artery disease. Heart Vessels. 2018;33:1275-1281.

21. Gur EB, Karadeniz M, Genc M, et al. Relationship between mean platelet volume and vitamin D deficiency in gestational diabetes mellitus. Arch Endocrionol Metab. 2015;59:448-454.
22. Cumhur CM, Cure E, Yuce S, Yazici T, Karakoyun I, Efe H. Mean platelet volume and vitamin D level. Ann Lab Med. 2014;34:98-103.

23. Harrison $\mathrm{P}$, Goodall $\mathrm{AH}$. Studies on mean platelet volume (MPV)-new editorial policy. Platelets. 2016;27:605-606.

24. Aihara A, Azuma H, Matsumoto T. Vitamin D-vitamin D receptor system regulates antithrombogenicity in vivo. Clin Calcium. 2006;16:1173-1179.

25. Silvagno F, De Vivo E, Attanasio A, Gallo V, Mazzucco G, Pescarmona G. Mitochondrial localization of vitamin $D$ receptor in human platelets and differentiated megakaryocytes. PLoS One. 2010;5:e8670.

26. Song LN. Demonstration of vitamin D receptor expression in a human megakaryoblastic leukemia cell line: regulation of vitamin $\mathrm{D}$ receptor $\mathrm{mRNA}$ expression and responsiveness by forskolin. J Steroid Biochem Mol Biol. 1996;57:265-274.

27. Shaik-Dasthagirisaheb YB, Varvara G, Murmura G, et al. Role of vitamins D, E, and C in immunity and inflamation. J Biol Regul Homeost Agents. 2013;27:291-295.

28. Singh U, Devaraj S, Jialal I. Vitamin E, oxidative stress, and inflammation. Annu Rev Nutr. 2005;25:151-174.

29. Bonaccio M, Di Castelnuovo A, De Curtis A, et al. Adherence to the Mediterranean diet is associated with lower platelet and leukocyte counts: results from the Moli-sani study. Blood. 2014;123:3037-3044.

30. Ishii T, Miyazawa M, Takanashi Y, et al. Genetically induced oxidative stress in mice causes thrombocytosis, splenomegaly and placental angiodysplasia that leads to recurrent abortion. Redox Biol. 2014;2:679-685.

31. Broudy VC, Lin NL, Fox N, Taga T, Saito M, Kaushansky K. Thrombopoietin stimulates colony-forming unitmegakaryocyte proliferation and megakaryocyte maturation independently of cytokines that signal through the gp130 receptor subunit. Blood. 1996;88:2026-2032.

32. Fu X, Wang XD, Mernitz $H$, Wallin $R$, Shea MK, Booth SL. 9-Cis retinoic acid reduces 1alpha,25dihydroxycholecalciferol-induced renal calcification by altering vitamin K-dependent gamma-carboxylation of matrix gamma-carboxyglutamic acid protein in $\mathrm{A} / \mathrm{J}$ male mice. J Nutr. 2008;138:2337-2341.

33. Di rosa M, Malaguarnera G, De Gregorio C, Palumbo M, Nunnari G, Malaguarnera L. Immuno-modulatory effects of vitamin D3 in human monocyte and macrophages. Cell Immunol. 2012;280:36-43. 